Polymer Journal, Vol. 25, No. 11, pp 1169-1174 (1993)

\title{
Copolymerization of Sodium 10-Undecenoate with Sodium 3-Allyloxypropionate
}

\author{
Kenichiro Arai and Kouji Yamazaki \\ Faculty of Engineering, Gunma University, Kiryu, Gunma 376, Japan
}

(Received April 1, 1993)

\begin{abstract}
The copolymerization of sodium 10-undecenoate (UANa) with sodium 3alloyoxypropionate (APNa) was examined under irradiation with UV light. The copolymerization rate was lower than homopolymerization rates of UANa and APNa. Each copolymer showed only one peak in the gel permeation chromatogram, which suggests that copolymerization really occurs, although the number-average degree of polymerization $\left(\overline{D P_{n}}\right)$ of the copolymers differed depending on composition of monomer feed in the copolymerization system. $\overline{D P_{n}}$ of the copolymer increased from 12 to 30 with APNa in the monomer feed. Reactivity ratios of the monomers were $r_{\mathrm{UANa}}=0.65 \pm 0.22$ and $r_{\mathrm{APNa}}=0.63 \pm 0.27$. Critical micelle concentration $(\mathrm{CMC})$ and pre-CMC of the copolymer, estimated from the UV absorption spectrum of Methylene Blue added to the aqueous solution of the copolymer, increased with APNa unit in the copolymer. The maximum amount of solubilized benzene into the aqueous solution of the copolymer increased with concentration of the copolymer higher than its pre-CMC, and decreased with increasing APNa unit in the copolymer.
\end{abstract}

KEY WORDS Copolymerization / Sodium 10-Undecenoate / Sodium 3-Allyloxypropionate / Monomer Reactivity Ratio / Degree of Polymerization / Maximum Amount of Solubilized Benzene / Critical Micelle Concentration /

Sodium 10-undecenoate $\left[\mathrm{CH}_{2}=\mathrm{CH}-\right.$ $\left(\mathrm{CH}_{2}\right)_{8}-\mathrm{COONa}, \mathrm{UANa}$, has been found to form micelles at concentrations higher than the pre-critical micelle concentration (pre-CMC) and polymerize effectively in the micelle form under irradiation with UV light ${ }^{1}$ or $\gamma$-ray. ${ }^{2-5}$ The obtained polymers had some interesting characteristics such as more pronounced hydrophobic domain than that of monomeric counterpart in water. ${ }^{6}$ We investigated catalysis systems in the presence of poly(UANa) and its derivatives, ${ }^{7}$ in which the polymer interacts hydrophobically with the substrate to accelerate catalysis. However, application of poly(UANa) to the catalysis as a catalyst is limited to alkali aqueous solution, and the numberaverage degree of polymerization $\left(\overline{D P_{n}}\right)$ of poly(UANa) is low as 12 as a polymer, reflecting association number of the micelle of
UANa. ${ }^{8}$ Thus we tried to copolymerize UANa with some comonomers to overcome these limitations. In preliminary experiments, we found that the copolymerization of UANa with other monomers was not so easy. For example, in the copolymerization of UANa with styrene solubilized into the UANa micelle in water, practically no copolymer was obtained.

In the present paper, to estimate the copolymerizability of UANa, we examined the copolymerization of UANa with sodium 3-allyloxypropionate $\left[\mathrm{CH}_{2}=\mathrm{CHCH}_{2}-\mathrm{O}-\right.$ $\left(\mathrm{CH}_{2}\right)_{2}-\mathrm{COONa}, \mathrm{APNa}$, which has a similar chemical structure to but a shorter aliphatic chain than UANa. Some properties of the obtained copolymers as surfactants are also examined. 


\section{EXPERIMENTAL}

\section{Reagents}

Water was deionized and distilled. All reagent grade chemicals including 10-undecenoic acid (Wako Pure Chemicals Ind., Tokyo) and 3-allyloxypropionic acid (Tokyo Kasei Chemicals Ind., Tokyo) commercially available were used without further purification, unless otherwise noted.

\section{Polymerization}

10-Undecenoic acid and 3-allyloxypropionic acid were neutralized with sodium hydroxide aqueous solution monitoring the $\mathrm{pH}$ with a $\mathrm{pH}$-meter to get aqueous solutions of UANa and APNa, respectively. A mixed solution of UANa and APNa at a given composition was irradiated with UV light from a high pressure mercury lamp (Toshiba $\mathrm{H}-400,400 \mathrm{~W}$ ) under nitrogen atmosphere at $40^{\circ} \mathrm{C}$ for a given time in a quartz reaction vessel. The reaction mixture was concentrated under vacuum to $1 / 10$ the volume and added to 20-times the volume of ethanol. The precipitate was filtered, washed with ethanol, and dried under vacuum.

The yields of polymers were determined gravimetrically, and compositions of the copolymers were estimated from the content of carboxylic acid group per unit weight determined by $\mathrm{pH}$ titration.

\section{Measurements}

Molecular weight distribution of the copolymers, converted to the free acid-form and dissolved in tetrahydrofuran to avoid formation of micelles according to the method previously reported, ${ }^{1}$ were determined by gel permeation chromatography (GPC: Apparatus, Toyo Soda Co., Ltd., Tokyo; HLC802UR: Column, TSK-G2000). The compositions of the copolymers quantitatively fractionated by GPC were also analyzed by $\mathrm{pH}$ titration.

The maximum amount of solubilized benzene into the aqueous solution of the copolymers was determined as follows: Benzene was added dropwise from a buret into an aqueous solution of the copolymer. The amount of benzene just before turbidity appeared, monitoring with a turbidimeter, was estimated as the maximum amount of solubilized benzene.

Critical micelle concentration (CMC) and pre-CMC were estimated with change in UV absorption spectrum of Methylene Blue added to the aqueous solution of the copolymer with the changing concentration.

UV absorption spectra were obtained with a Hitachi Spectrometer 124 (Hitachi Seisakusho Co., Tokyo).

\section{RESULTS AND DISCUSSION}

Copolymerization of UANa with APNa at different compositions was examined and compared with homopolymerizations of UANa and APNa. Figure 1 shows typical changes in yields of polymers in copolymerization and homopolymerizations under irradiation with UV light, with irradiation time. The yield of the copolymer (in the case shown in

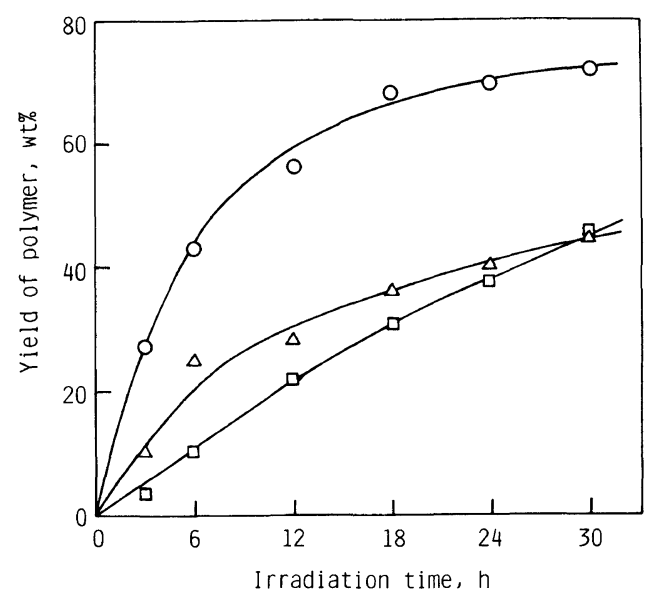

Figure 1. Changes in yields of polymers obtained in the copolymerization of sodium 10-undecenoate (UANa) with sodium 3-allyloxypropionate (APNa) $(1: 1 \mathrm{in} \mathrm{mol} \mathrm{in}$ monomer feed, $\square$ ) and homopolymerization of UANa $(\triangle)$ and APNa $(O)$ under irradiation with UV light, with irradiation time. [Monomer] is constant at $0.12 \mathrm{~mol} \mathrm{l}^{-1}$. 


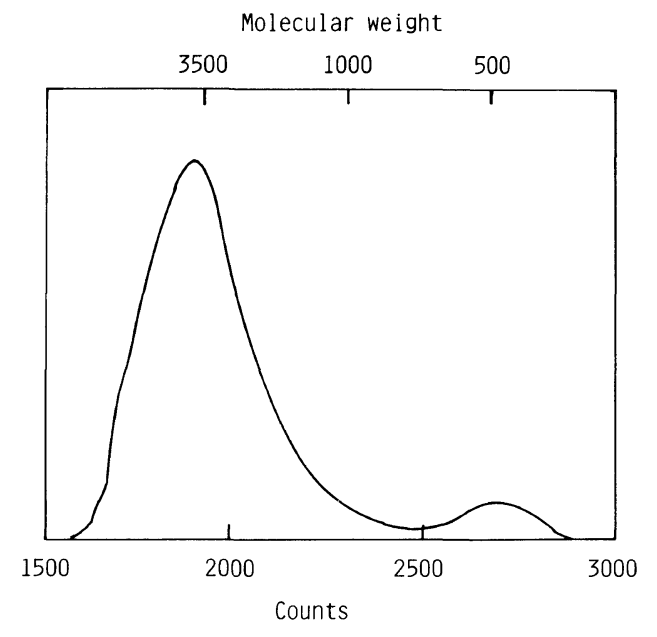

Figure 2. Typical gel permeation chromatogram of a copolymer. [UANa] and [APNa] in monomer feed are $0.06 \mathrm{~mol} \mathrm{l}^{-1}$. Irradiation time, $18 \mathrm{~h}$.

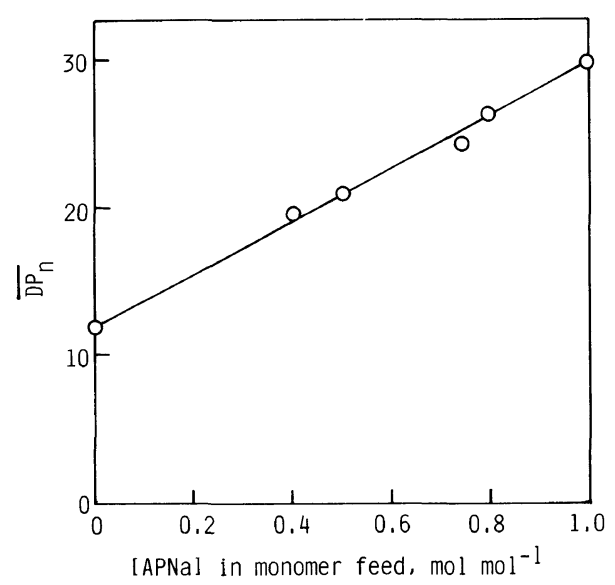

Figure 3. Change in number-average degree of polymerization $\left(\overline{D P_{n}}\right)$ of copolymer, with changing amount of $\mathrm{APNa}$ in monomer feed. Irradiation time, $18 \mathrm{~h}$.

Figure 1, UANa and APNa in the monomer feed is $1: 1$ in mol) was lower than those of both homopolymers, at least in the initial reaction range.

Molecular weight distribution of the copolymers were examined by GPC. Figure 2 shows a GPC chart of a copolymer. Only one peak was obtained, which may indicate the formation of the copolymer of UANa with APNa, although a small peak was found in a lower

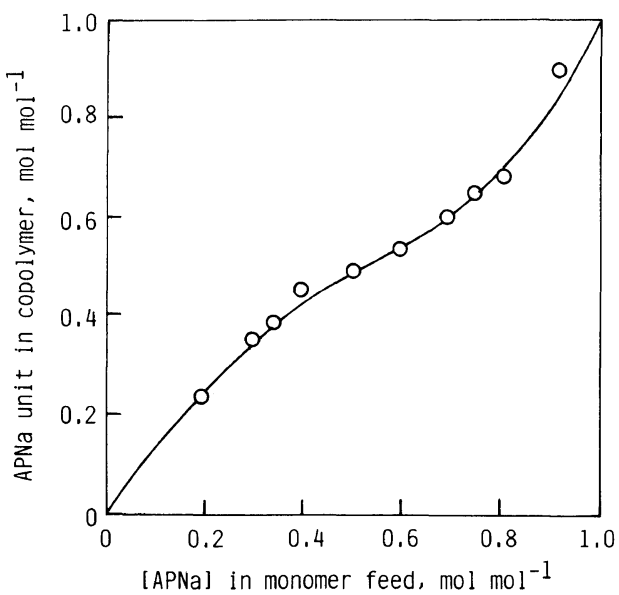

Figure 4. Plot of APNa unit in the copolymer against amount of APNa in monomer feed. Yields of copolymers were limited below $5 \mathrm{wt} \%$ with changing irradiation time.

molecular weight range, probably due to imperfect purification. Number-average degree of polymerization $\left(\overline{D P_{n}}\right)$ of the copolymers was calculated from GPC data and compositions of the copolymers fractionated, and plotted against APNa in the monomer feed for copolymerization in Figure 3. $\overline{D P_{n}}$ increased from 12 to 30 with APNa.

To investigate quantitatively the copolymerizability of UANa with APNa, monomer reactivity ratios were estimated. Figure 4 shows a plot of APNa unit in the obtained copolymer against APNa in the monomer feed, where copolymer yield was limited below $5 \mathrm{wt} \%$ with changing reaction time. In the Fineman-Ross plot $^{9}$ of the data, a fairly good straight line was obtained (correlation coefficient, 0.954) and reactivity ratios were estimated as $r_{\mathrm{UANa}}=$ 0.65 (error for $95 \%$ confidence limit: \pm 0.22 ) and $r_{\mathrm{APNa}}=0.63 \pm 0.27$. The low accuracy of the values might result from the low DP of the copolymer.

CMC and pre-CMC of the obtained copolymer in the aqueous solution were examined with change in UV absorption spectrum of Methylene Blue added to the solution with the changing concentration. Figure 5 shows typical changes in the spectrum. Usually, the shift of 


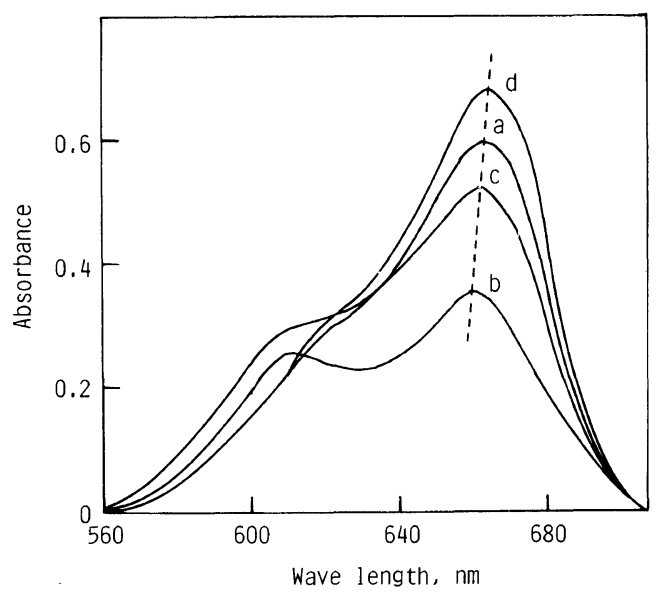

Figure 5. UV absorption spectra of Methylene Blue added to UANa-APNa copolymer (UANa unit, 70.2 mol\%) aqueous solution at various concentrations. [Copolymer], mol $1^{-1}$ as total monomer unit: a, $0 ; \mathrm{b}$, 0.00057 ; c, 0.0057 ; d, 0.057 .

a peak in the spectrum with changing concentration of a surfactant is used to estimated CMC. ${ }^{6}$ However, in the figure a peak around $660 \mathrm{~nm}$ only slightly shifted, whereas the peak height considerably changed, although the peak shift and change in the peak height were parallel to each other. The blue shift corresponds to decrease in peak height and the red shift to increase in peak height. Accordingly, we estimated the CMC and preCMC using change in peak height of the absorption around $660 \mathrm{~nm}$. Figure 6 shows typical change in the peak height with changing concentration of the copolymer. Peak height decreased with increasing concentration of the copolymer in the very low concentration range, showed a minimum, then increased, and leveled-off in the still higher concentration range. The decrease in the peak height in the very low concentration range of the copolymer is inferred to result from electrostatic interaction of Methylene Blue molecules with copolymer molecules. The increase in peak height at concentrations higher than that corresponding to the minimum is assumed to reflect that the atmosphere around the Methylene Blue

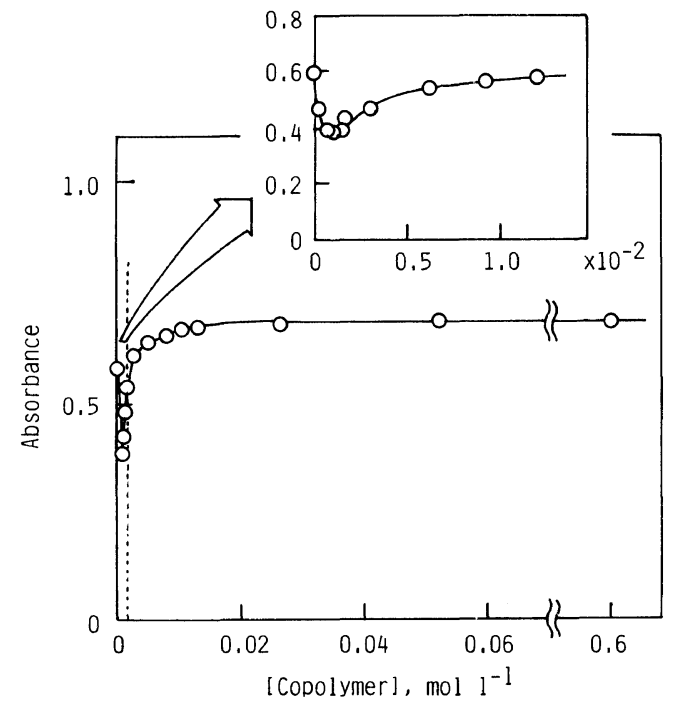

Figure 6. Typical change in UV absorption peak height of Methylene Blue around $660 \mathrm{~nm}$ with changing concentration of copolymer (UANa unit, $70.2 \mathrm{~mol} \%$ ). [Copolymer] refers to total monomer unit.

molecules becomes more hydrophobic with the formation of preliminary micelles. Leveling-off of the increase in the peak height may indicate that hydrophobicity of the atmosphere around Methylene Blue molecules becomes constant with the formation of stable micelles of the copolymer. Thus, the concentrations corresponding to the minimum and at which peak height begins to be constant may be estimated as pre-CMC and $\mathrm{CMC}$, respectively. The values of poly(UANa) are slightly different from those estimated by the fluorescence method using trimethyl-1-pyrenylmethylammonium iodide as a probe. ${ }^{6}$

Figure 7 shows plots of the pre-CMC and $\mathrm{CMC}$ against APNa unit in the copolymer for some copolymers. Pre-CMC and CMC increased slightly with increasing APNa unit in the copolymer at the amounts of APNa unit less than $50 \mathrm{~mol} \%$, but they did so steeply at the more amounts. The steep increase in the pre-CMC and $\mathrm{CMC}$ may reflect that lowering of hydrophobicity of the copolymer with increasing APNa unit is accelerated at the more amounts, although change in DP of the co- 


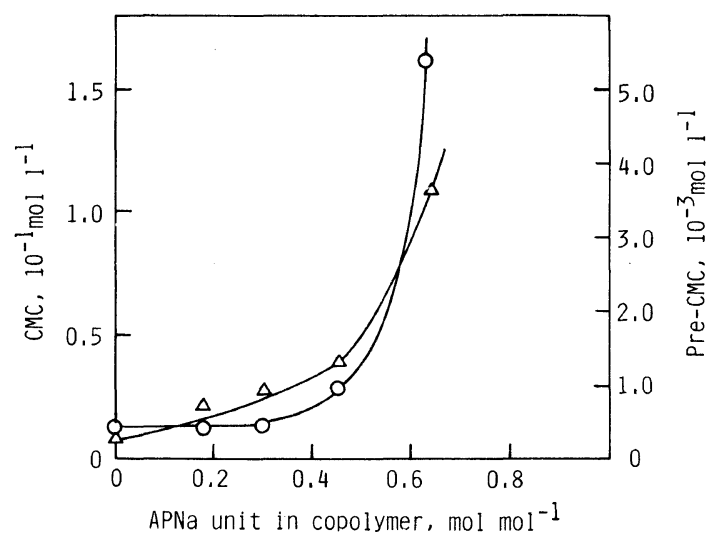

Figure 7. Plots of pre-CMC $(\triangle)$ and CMC $(\bigcirc)$ of copolymer in aqueous solution, estimated with change in absorption spectrum of Methylene Blue, against amount of APNa unit in copolymer.

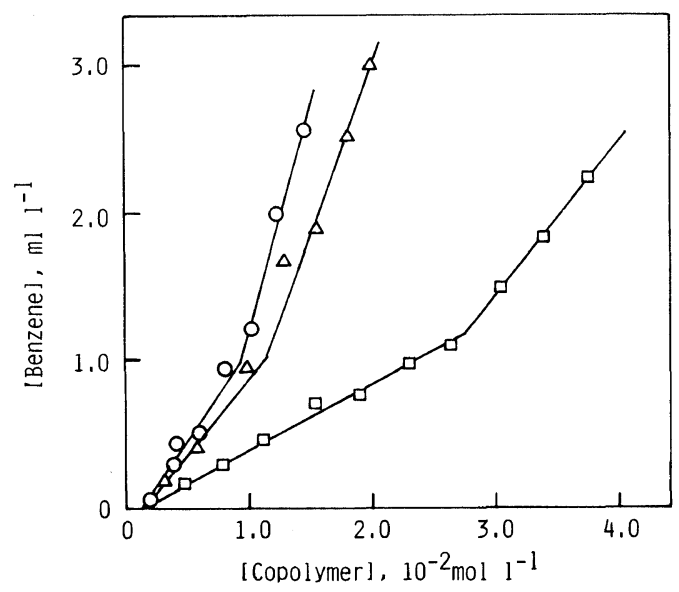

Figure 8. Plot of maximum amount of solubilized benzene into aqueous solution of copolymers with different compositions against concentration of the copolymer. APNa unit in copolymer in mol\%: $\bigcirc, 0 ; \triangle, 35.3 ; \square, 46.4$. [Copolymer] refers to total monomer unit.

polymer may also influence these changes.

Figure 8 shows plots of the maximum amount of solubilized benzene into the aqueous solution of the copolymers with different compositions against the concentration of copolymer. Benzene is not solubilized at very low concentration of copolymer, but it is solubilized at the concentrations higher than a certain concentration and the amount of solubilized benzene increases proportionally with concentration of the copolymer. Each plot shows two straight lines which have a break point at a certain concentration (in the previous paper $^{1}$ we could not find the break point because we investigated change in a much higher concentration range of the polymer). The concentrations at which solubilization of benzene begins with increasing concentration of the copolymer and which correspons to the break point of the plot approximately coincided with the pre-CMC and the CMC, respectively, estimated with change in the spectrum of Methylene Blue.

The above behaviors may result from the formation of preliminary and genuine micelles with increasing concentration of copolymer. Mechanism of micelle formation is not clear at present. The amount of solubilized benzene at the same concentrations of different copolymers decreased with increasing APNa unit in the copolymer. For copolymers containing the APNa unit at more than $50 \mathrm{~mol} \%$, a little benzene was solubilized into the aqueous solution, but the break point in the plot of the amount of solubilized benzene against the concentration of the copolymer was not clear. These results agree well with changes in pre-CMC and CMC with increasing APNa unit in the copolymer.

\section{CONCLUSION}

Copolymerization of UANa with APNa was investigated and copolymerization was confirmed to occur from the fact that only one peak was found in the GPC chart of the copolymer. $\overline{D P_{n}}$ of the obtained copolymer increased from 12 to 30 with increasing APNa in the monomer feed. The reactivity ratios of the monomers were $r_{\mathrm{UANa}}=0.65 \pm 0.22$ and $r_{\mathrm{APNa}}=0.63 \pm 0.27$.

Pre-CMC and CMC, and the maximum amount of solubilized benzene into the aqueous solution of the copolymer were examined. Hydrophobicity of the copolymer was considerably reduced with increasing amount of 
APNa unit in the copolymer at more than $50 \mathrm{~mol} \%$, but not so much at less than this amount.

From above results, it is concluded that $\overline{D P_{n}}$ of poly(UANa) slightly increased by copolymerization with APNa at less than $50 \mathrm{~mol} \%$ without much loss of hydrophobicity of the polymer.

Acknowledgment. We are grateful to $\mathrm{Mr}$. Akinobu Ono (Fujikura Co., Ltd.) for his helpful assistance in the present work.

\section{REFERENCES}

1. K. Arai, J. Sugita, and Y. Ogiwara, Makromol.
Chem., 188, 2511 (1987).

2. C. E. Larrabee, Jr. and E. D. Sprague, J. Polym. Sci., Polym. Lett. Ed., 17, 749 (1979).

3. E. D. Sprague, D. C. Duecker, and C. E. Larrabee, Jr., J. Am. Chem. Soc., 103, 6797 (1981).

4. C. M. Paleos, S. Voliotis, and G. MargomenouLeonidopoulou, J. Polym. Sci., Polym. Chem. Ed., 18, 3463 (1980).

5. C. M. Paleos, P. Dais, and A. Malliaris, J. Polym. Sci., Polym. Chem. Ed., 22, 3383 (1984).

6. K. Arai, Y. Maseki, and Y. Ogiwara, Makromol. Chem., Rapid Commun., 8, 563 (1987).

7. K. Arai and T. Okabe, Polym. J., 24, 769 (1992).

8. K. Arai, S. Miyahara, and T. Okabe, Makromol. Chem., 192, 2183 (1991).

9. M. Fineman and S. D. Ross, J. Polym. Sci., 5, 269 (1950). 